\title{
ASSESSMENT AND MEASUREMENT OF VITAL SIGNS OF HUMAN BEINGS USING IOT ARCHITECTURE
}

R. Raja Sudharsan

Department of Electronics and Communication Engineering, School of Electronics and Electrical Technology, Kalasalingam Academy of Research and Education, Krishnankoil, Virudhunagar (Dt), (India).

E-mail: rajasudharsan@klu.ac.in ORCID: https://orcid.org/0000-0001-8844-9774

\section{Citación sugerida:}

Sudharsan, R. R. (2021). Assessment and measurement of vital signs of human beings using IoT architecture. 3C Tecnología. Glosas de innovación aplicadas a la pyme, Edición Especial, (noviembre, 2021), 491-511. https://doi.org/10.17993/3ctecno.2021.specialissue8.491-511 


\section{ABSTRACT}

The concept and architecture of Internet of Things (IoT) is getting progresses every day. This is proposed to various objects globally which is accessible from the internet and gets integrated to many different portable devices. This Paper presents the four-layer architecture of communication protocols in which it is oriented to medical applications. Different portable devices (Sensors) of medical application have been used for measuring vital signs (Temperature, Blood Pressure, Respiration rate, Pulse rate and Electrocardiogram, etc.,) of human body, these sensors will give series of information or data about the vital signs measured. This Measured data (information) is transmitted to Android based mobile application (Mobile App) through which the data can be monitored via Bluetooth (IEEE 802.15.1) at 2.4 GHz and the device is controlled by the single board computers. The Middleware services for maintaining (Collecting and retrieving) of databases in the server are studied.

\section{KEYWORDS}

Internet of Things (IoT), Four-layer Architecture, Vital Signs, Android Mobile Application, Middleware Services. 


\section{INTRODUCTION}

The importance of Internet of Things (IoT) has become vital to the society, since its rapid growth in the Internet technologies and communication systems. In the current scenario, we get access to internet via Smart Mobile phones, Computers, Tablets, Televisions etc., at anytime and anywhere. In the similar fashion, the Internet of Things (IoT) is being developed which is not used only "human" but also "things". It is used various fields of applications such as the Home Appliances, Agriculture, Medical Devices and so on. The internet is applied in various fields, it conveys an idea in which the "things" is well communicated and interconnected or interrelated with different solutions. Mainly, internet for communication to other devices, over the cloud and some field of services (events, database, semantic, service based and application specific). This IoT can communicate with various sources developed. They are, Bluetooth, Wi-fi, Zigbee, Radio frequency Identification, Near Field Communication, Lo-Ra etc., these mediums are used depending on the application requirement.

It may be Short-range, long range, low power, high data rate, security (secured data transfer) and also type of portable devices (Sensors and Actuators) used in the application. For very long range and higher bandwidth "TV Whitespace" can be used for data transfer, even the target device is very far away from the source operating device. In other-words, this IoT denotes the worldwide network connections and its addressed devices. According to Gubbi et al. (2013), the IoT connects to various sensors and actuators components, providing the ability to them for transmitting the information from one host to other host using various mediums. This will lead database collection and management, big data analytics, cloud computing, etc.

It is may be contemplated that evolution of Internet of Things (IoT) will plays a vital role in the near future. So, more investments have been made in the Research and Development, thus, public and private investments have been made in R\&D, Prototype demonstration and various deployment activities. There are various architectures and communication technologies have also been emerged since last decade for performing the portable devices and its communication to server using various resources. 
In this paper, we present an architecture for a Health Care Application, taking into considerations of Various sensor (portable devices), Protocols, Communication medium along with the study of Middleware services. In SECTION-II, related literature survey was discussed. In SECTION-III, four-layer architecture has been discussed along with its Protocols and Portable devices. In SECTION-IV, studied the various Middleware Services and in the SECTION-V, the results have analyzed and discussed. Finally, this paper is concluded with a Conclusion in the SEGTION-VI.

\section{RELATED WORK}

Fatmi, Hussain and Al-Rubaie (2017) proposed a remote monitoring system in which a mobile app running on a smartphone platform reads patients' vital signs such as blood pressure signal, blood saturated oxygen ( $\mathrm{SpO} 2)$, and temperature using single board computers. The app transports the data to a server located in a cloud or to a corporate data centre. Misbahuddin et al. (2018) proposed a patient monitoring system in which the patient's vital signs (e.g) ECG, temperature, etc., are measured using the wearable sensors. This information is transferred to the Doctor's Server/database using Internet of Things. (i.e) transferring the patient's vital signs via ESP8266 to the Server/Database.

Ren et al. (2010) proposed a mobile healthcare system. In which the EEG, ECG, Pulse and blood pressure are measured using wearable sensors and transfer those measurements to mobile phones. Patients can measure their vital sign round the clock and get to know the conditions of their Body and also can prescribe medicines from doctors accordingly. These data are made secured and privacy from various attacks. This system enhances the secured data and telemedicine systems.

Banuleasa et al. (2016) proposes the design of a wearable electronic system, which is independent, aggregates and processes data from a diverse network of sensors that measure the body's vital signs and aggregate that data into a microcontroller driven platform, which is connected to a remote gateway for uploading the data. The remote gateway will be the subject's smartphone that keeps alive the communication through GPRS (General Packet Radio Service) to an internet available server or cloud service. Both the electronic system 
and the remote service (server/cloud) that receives the data, in the end, can generate social alerts and initiate various actions, server side, gateway side or even IOT device side.

Swaroop et al. (2019) proposed the design of a real-time health monitoring system which can store a patient's basic health parameters (vital signs parameters). The data can be made available to a medical practitioner as an alert and for monitoring by multiple means of communication. The data from the health monitoring system transfers to doctors or any medical practioner's server using multi node communication. (i.e) transfer the data using Bluetooth (BLE), sensing SMS (messaging services) using GSM module and though internet using Wifi, which enhances the multimode communication for health care systems.

Prakash, Girish \& Ganesh (2016), in their work, propose a human vital sign monitoring system which includes measurement of pulse rate and body temperature of patients using wearable sensors from remote locations. The network processor CC3100 and Computational processor MSP430 is interfaced with sensory components and made to communicate the emergency signals through Global System for Mobile (GSM) like messaging service and SMTP server. This Simple Mail Transfer Protocol (SMTP) server can be achieved by implementing a TCP/IP stack and Ethernet interface in a MSP430 controller.

\section{MATERIALS AND METHODS}

There is no single agreement on architecture for IoT, which is accepted universally. Different architecture has been proposed by several researchers. There are two main architectures, they are: Three layer and Five-layer architecture. Among these, three-layer architecture is more basic one which has perception layer, network layer and application layer. This Perception layer is commonly known as Physical layer. In which, it has sensors for sensing the information and gathering it related to the application environment. In other-words, the sensors will sense some physical parameters. The network layer is responsible for connecting to smart devices (Single board computers) as a gateway. It adds the features, that it can transmits the data and process the data from the sensors in the physical layer. The final layer will be the Application layer, in this layer it delivers the application explicit administrations to the client (users). Three-layer architecture has been overcome by the fivelayer architecture since it focuses on only few aspects of Internet of Things (IoT). 
The Five Layer architecture has the additional layers such as transport layers processing layer and business layers being both Perception and Application layers are common for both Three and Five layer. This (i) Transport layer will send the Sensors data to Processing layer from the perception layer using Wireless via Bluetooth, Wifi, LAN, NFG, RFID etc., (ii) The Processing Layer will do the job of Middleware services. This Middleware services will Manage, store, analyze and process very huge amount of data from the transport layer. It employs various databases, Big Data Analytics and Cloud Computing. (iii) The Business layer which included the entire Internet of things (IoT) module, Profit models and User's privacy. In a classic social IoT setting, we treat the gadgets and services as bots where they can set up connections among them and adjust them after some time. This will enable us to consistently give the gadgets a chance to collaborate among one another and accomplish an intricate undertaking.

To make such a model work, we need numerous interoperating parts. 1) Require a "Unique technique of object Identification". (i.e.) an ID has been assigned to every device or object based on the Classical parameters namely: UUID, MAC ID, product code or other custom method for object identification. 2) The second will be the "Metainformation" which comes along with ID of a device/object. The Metainformation will gives the description of the device's development and operation. 3) After Metainformation, "Security Control" plays a vital role in object communication which is much similar to "Friend list" setting in Facebook (Social Application). 4) Next is "Service Discovery", similar to service cloud in which have to store the data in the dedicated directories commonly termed as Database Management System (DBMS). It is very important for devices to keep up-to-date information/data for future services. 5) The final part will be the "Service Configuration", the goal of this part is to provide better integration, services to new users.

Based on these different Components, the IoT architecture are classified based on the communication network (Personal Area Network (PAN), Local Area Network (LAN), Wide Area Network (WAN), Metropolitan Area Network (MAN) etc.). In general, the IoT architectures will have set of protocol layers differs majorly on the applications (Home appliances, Medical, agriculture etc.,). These layers can be related to Internet Protocol (IP) layers. There are totally four layers in which the complete set of devices can be ready, they are: Sensing layer (Physical layer), Network Layer (Gateway layer), Transport layer 
(Management Layer) and Application layer (Data Server). These layers follow a set of protocols to execute the necessities.

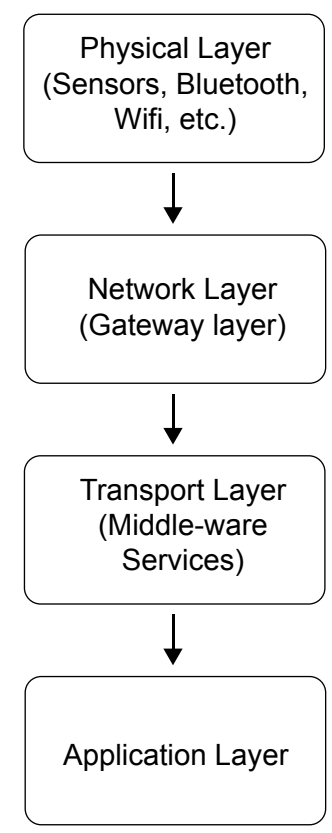

Figure 1. Four Layer Architecture.

Source: own elaboration.

\subsection{PHYSICAL LAYER}

This layer which contains different sensors for measuring major vital signs of Human Body. This Sensors will measure the parameters and gathers information about the vital signs. The major vital signs measured is temperature, blood pressure, Electrocardiogram (EGG) and Electromyogram (EMG) from a human body using the corresponding sensors such as (i) DS18B20 (water proof) by Maxim Integrated - This sensor will measure human body temperature which has the accuracy of about $0.50 \mathrm{C}(-10-1000 \mathrm{C})$, operating temperature ranges from $-550 \mathrm{C}$ to $1250 \mathrm{C}$ and operating voltage is 3.0 to $5.5 \mathrm{~V}$.

Moreover, this sensor is Water proof. (ii) AD8232 module by SparkFun electronics - This sensor will measure Electrocardiogram (ECG) of human being, which has supply voltage of $2.0 \mathrm{~V}$ to $3.5 \mathrm{~V}$, operating temperature is about $-650 \mathrm{C}$ to $1250 \mathrm{C}$, Gain Error is $0.4 \%$, CMRR is 80 dB. (iii) MyoWare Muscle Sensor by SparkFun Electronics - This sensor will measure the Electromyogram of a human being, which has the operating voltage of about 
2.0 $\mathrm{V}$ to $5.7 \mathrm{~V}, \mathrm{CMRR}$ is $85 \mathrm{~dB}$, Gain is 1000 and bandwidth of $25 \mathrm{~Hz}$ to $480 \mathrm{~Hz}$ and (iv) ASDXACX015PAAA5 by Honeywell - This sensor measures the Blood Pressure of a human body, which has the accuracy deviation of about $1.5 \%$, operating voltage of 3.3 $\mathrm{V}$ to $5.0 \mathrm{~V}$, operating temperature is about $00 \mathrm{C}$ to $850 \mathrm{C}$ and it has the response time of $1 \mathrm{~ms}$. These sensors measure the corresponding Physical parameters and identifies the smart objects such as low power embedded devices with more security control, low power and high data rate (Proper Gateway) in order to transfer the gathered information.

\subsection{NETWORK LAYER (GATEWAY)}

This Layer will act as a gateway, in which the data from physical layer can be transmitted to the network layer by various medium such as Bluetooth, Wi-fi, Zigbee, Radio frequency Identification, Near Field Communication, Wireless Sensor Networks to the Single Board Computers. Here, the data from the sensors are transmitted via Bluetooth (HC-05/ HC06) to the Android based Mobile Application. This data can also be transmitted to the single board Computer via Wifi module (ESP 8266 or Node MCU). The Internet Protocol version $4\left(\mathrm{IPv}_{\mathrm{v}}\right)$ is used as the main protocol for Network layer. It transmits the packets (Data) from host to host (i.e.) transmits data from source to destination. This achieved by using logical address (32 bits of integral values). The IPv4 is a connectionless protocol and used for packet switched networks. It operates on best effort model delivery model, but it does not assure a proper sequencing of data. This aspect includes the will leads to Data Integrity which is addressed to the next layer named Transmission Control Protocol in the Transport layer.

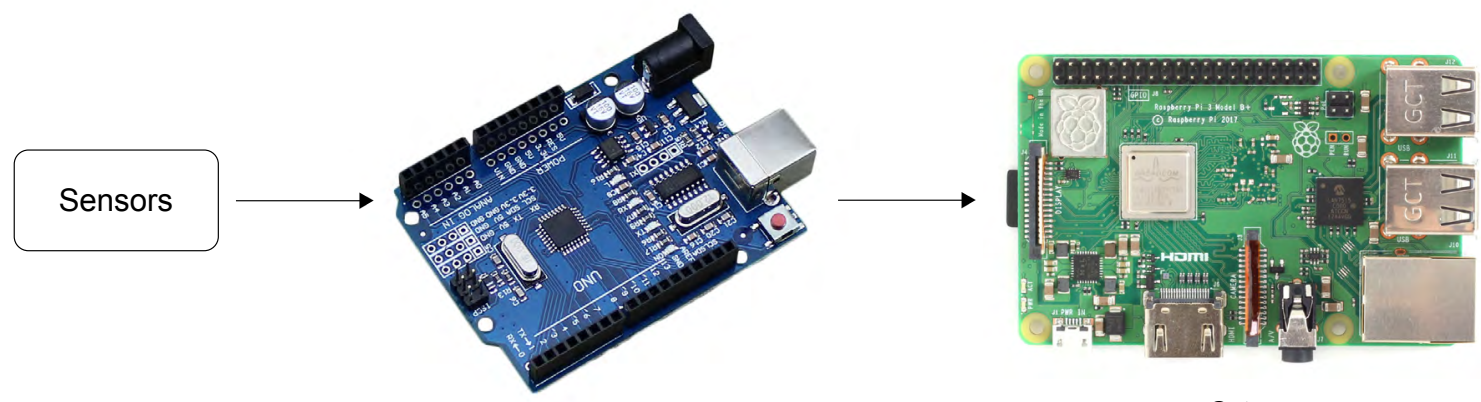

Gateway

Figure 2. Controller to Gateway Communication.

Source: own elaboration. 


\subsection{TRANSPORT LAYER}

This layer uses Transmission Control Protocol for data the data to be transported from network layer to the Application layer. The TCP defines the establish a data and maintain a network communication which application layer programs can exchange data (packets). The TCP has maximum of 60 bytes of data sequence. The main concept of TCP such as Byte Streaming, Connection oriented, Full duplex, Piggybacking, Error Control, Flow Control, and Congestion Control. The data from the Network layer is given to TCP layer, using IPv4 the data is sent to the application layer

i. Byte Streaming: This Byte Streaming is one of the major advantages of Transmission Control Protocol in which the data from Application layer to transport layer and vice versa send continuously without any losses of data (packets) in array format. The Data will be in terms of bytes (Collection of bits) which altogether form a segment

ii. Connection Oriented: This protocol is a Connection oriented unlike Internet Protocol (IP) which is connectionless. The purpose of connection oriented is reliability (added feature). In other words, the data loss or packets loss is very less while transmitting and receiving the data (packets). If the data loss is predicted, then the same data (packet) is retransmitted to the receiver. Whereas, in User Data Protocol (UDP) the reliability was not guaranteed. In TCP, 3-handshaking protocols takes places. They are: 1) Request, 2) Response and 3) send data (data transmission).

iii. Full Duplex: As known, the full duplex, will send and receive data from one device to other devices at the same time which is an added a feature of TCP.

iv. Piggybacking: It is similar and continuity of handshaking protocol. In which the data send to the receiver, the receiver sends the acknowledge that the full data (packet) are received without data (packet) loss. Once the Acknowledge receives to sender, it transmits another segment of data to receiver. Two steps of Sliding Window Protocol used, namely: "Go Back" and "Selective Repeat".

I. Go-Back: It retransmits all the frames in which alteast any one of the frames is damaged or lost. 
II. Selective Repeat: It retransmits that only frame which is damaged or lost.

v. Flow, Error and Congestion Control: This flow, error and congestion control mechanisms plays a vital in transmitting a from sender to receiver because the flow control is used to restrict the amount of data (packets) that the sender can send before waiting for acknowledgment and it (Flow Control) is based on two categories. They are feedback-based and Rate-based flow control. Whereas, the Error Control ensures the reliable communication of message by providing the sender with some positive and negative feedback or Acknowledgment about what is happening in the other end (receiver). In case of congestion, decides the capability of data (packets) can be sent at a time in a same network. In other words, if infinite data (packet) are sent at a time in a single network, that leads to congestion. These actions can be controlled in Transmission Control Protocol (TCP) of Transport Layer (TL).

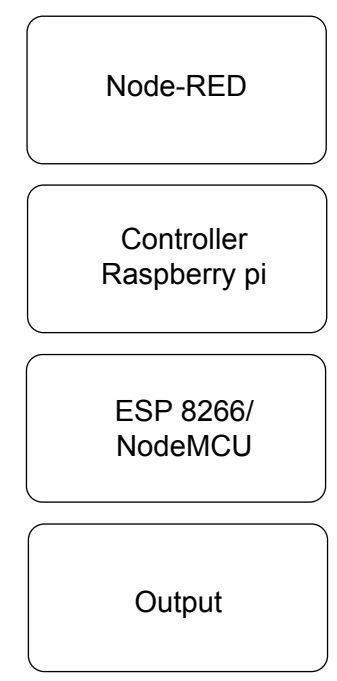

Figure 3. Flow of MQTT protocol.

Source: own elaboration.

\subsection{MIDDLEWARE SERVICES}

The Middleware Services provides interconnectivity to all the devices around us. In other words, the omni-present computing is the core of IoT (Internet of Things). The Middleware services is mainly used for different requirements of varied applications in order to make it more standard. This service will act as a software bridge between things and applications. In 
outline, the middleware conceptualizes the hardware and provides an integration between the hardware and application which is commonly termed as Application Programming Interface (API). This API, not only used for communication but also database management, Security control and privacy. In general, the middleware services are based on their design.

They are:

(A) Event Based: in event based, all the elements of device or machines can communicate with each other through different events. Each and every event has a type and few parameters. This Events are generated or produced by producers and received by consumers. This is commonly considered as a Publish/subscribe structure, which is depicted in the Figure 3.

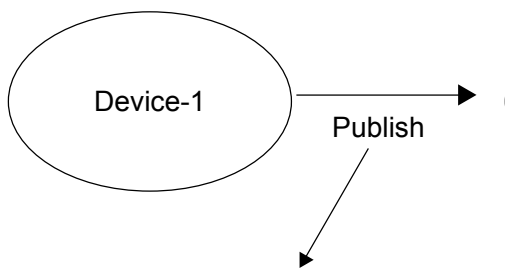

Device-1 sends (publish) a message

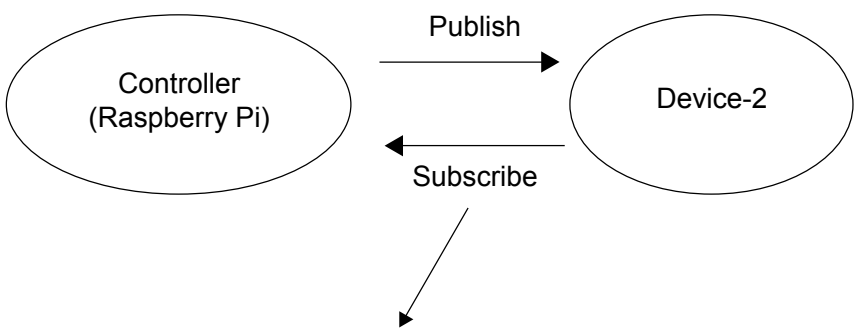

Device-2 subscribes the same message in which the same messages publishes in Device-1 Device- 2 receives the message

Figure 4. Publish/Subscribe Event.

Source: own elaboration.

(B) Service Based: the Service based Middleware which is mainly a kind of Service Oriented Architecture (SOA) that support for networked services. The important feature of SOA is that offerings are unbiased entities, with well-defined interfaces, which can be invoked in a general manner, without requiring the consumer to have expertise about how the service actually plays its responsibilities. This SOA rely on three main components. They are: (i) Service Registry, (ii) Service Provider and (iii) Service Consumer. This Service based middleware is wholly responsible enabling and development of services for these three conceptual key components. 


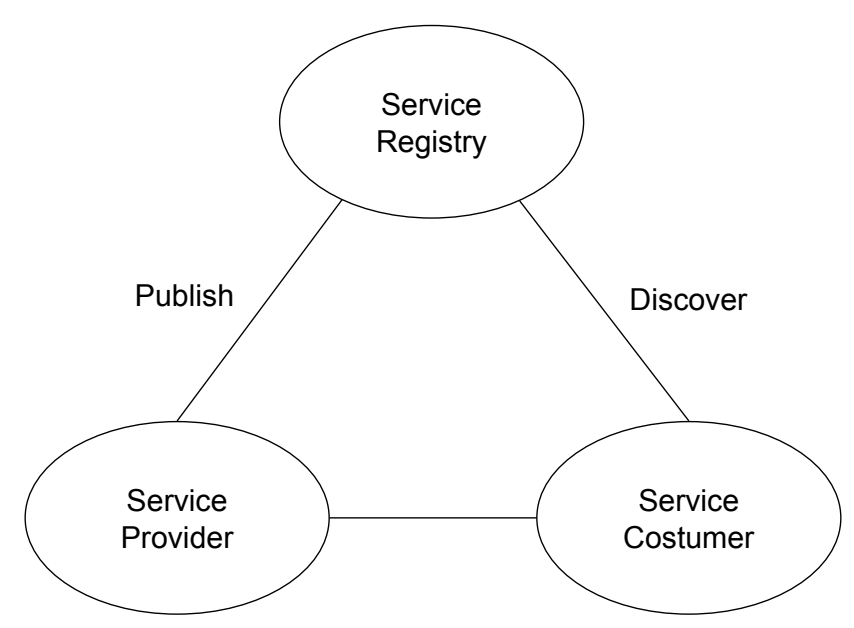

Figure 5. Three key parts of SOA.

Source: own elaboration.

(C) Database Based: in this method, the network of IoT systems/devices is considered as a digital relational database system. The database can then be queried by using the packages the usage of a query language. There is smooth to apply interfaces for extracting the records of data from the database. This approach has issues with scaling due to its centralized model.

(D) Semantic Based: this Semantic based middleware services are focused on interconnection of different types of systems or devices, in which it can communicate with various forms of data in the database. For this type of action, a separate framework has been used. In the case of common format of data, this middleware uses $\mathrm{N}$ adapters for $\mathrm{N}$ amount of data of same format in order, that the adapter to map $\mathrm{N}$ number of standards. In other words, the semantic based middleware services are used for mapping each resource with software layers for those corresponding resources. This software layers then communicate with each other by using their language which is based on semantic web.

(E) Application Specific: this application specific middleware services are mainly used for corresponding application which developed based on the whole architecture in the application layer. It is more coupled with the application layer. Comparing to other middleware services, this application specific middleware is not general purpose. 


\section{RESULTS AND DISCUSSION}

As the sensors are fixed to the human body in their respective location in order to measure its physical parameter. The sensor data are transferred from Physical layer to the mobile app using Bluetooth (i.e.) the physical parameters are measured by using the corresponding sensors and monitored using the mobile app (Android Application) as discussed in SECTION II. The Bluetooth module used here is HC-05/HC-06. This HC-05 send data using Serial port Protocol (SSP) at 2.4 GHz, low power, with Enhanced data rate. The hardware basic setup for the health care system for vital signs of human body is illustrated in the Figure 6.

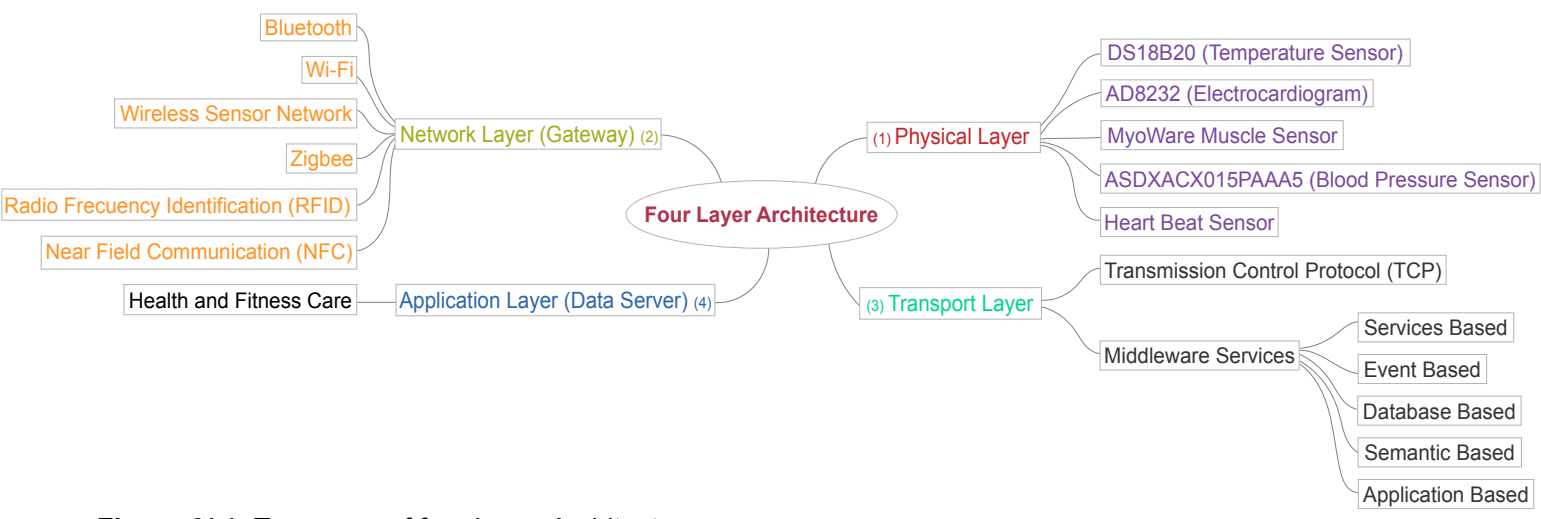

Figure 6(a). Taxonomy of four Layer Architecture.

Source: own elaboration.

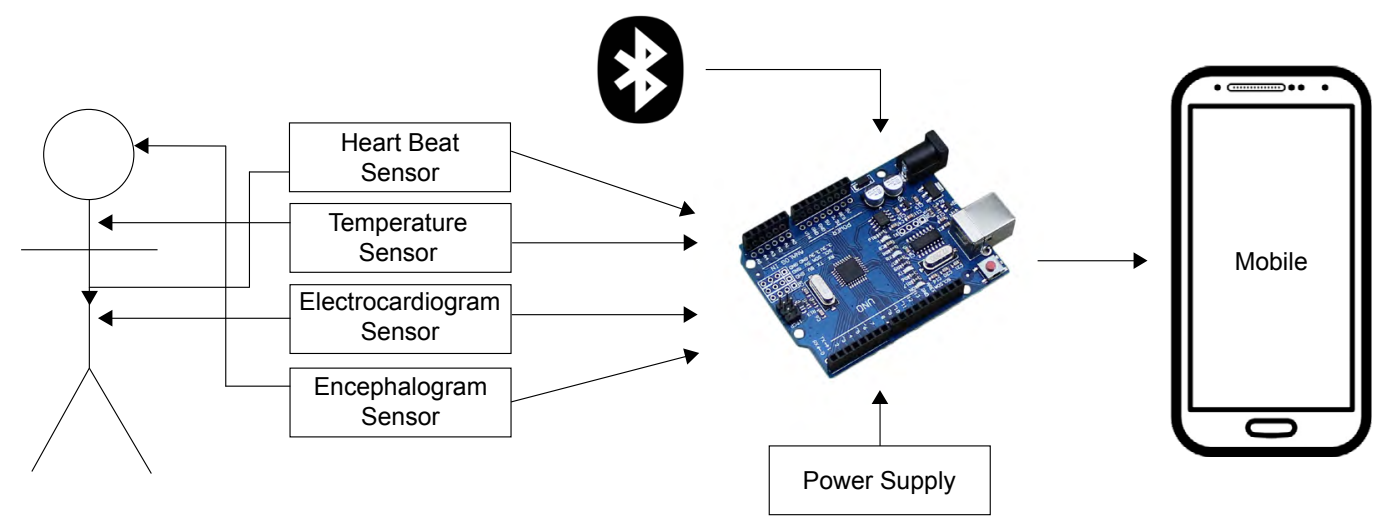

Figure 6(b). Hardware setup of health care system for Vital Signs.

Source: own elaboration. 


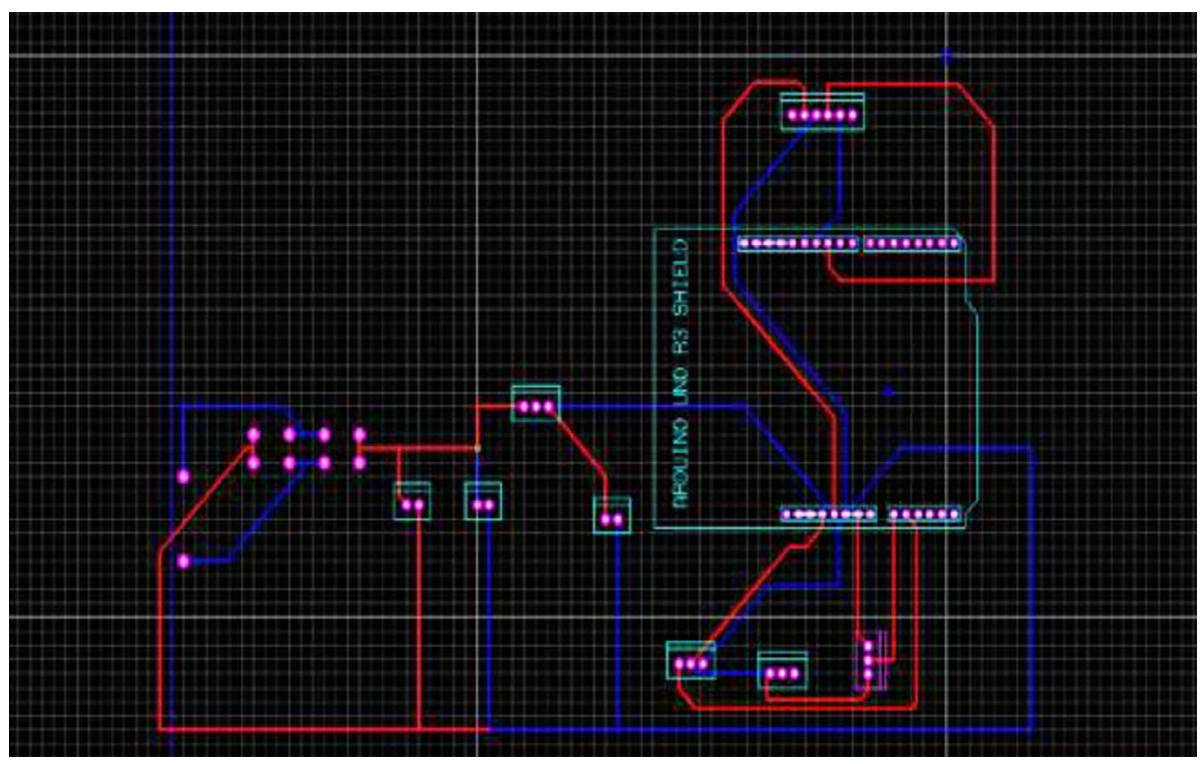

Figure 7. Layout of the device (Health Care System).

Source: own elaboration.

This system is controlled by a Single board computer (Arduino (Uno, Mega, Nano)/ Raspberry $\mathrm{Pi})$, in which the power supply given to the system is $5 \mathrm{~V}(2.5 \mathrm{~A})$.

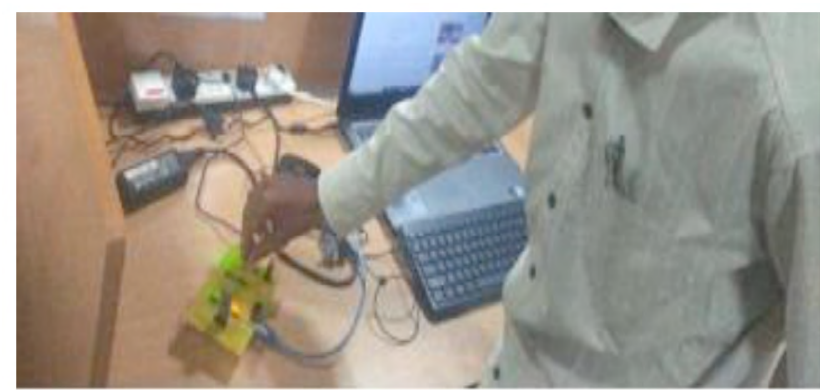

Figure 8. A Patient measuring his Temperature.

Source: own elaboration.

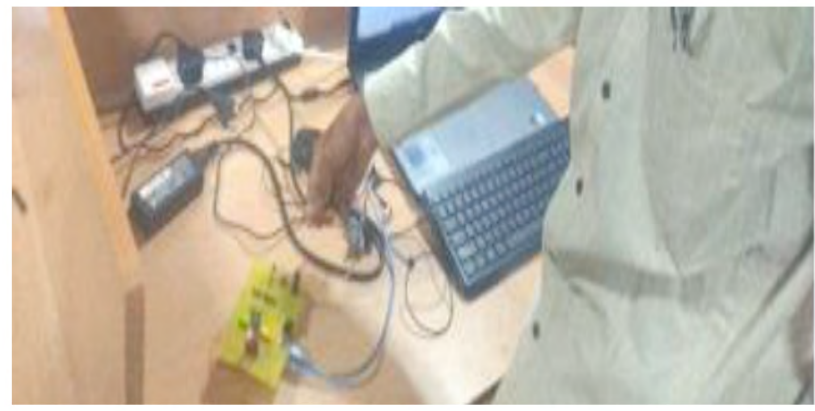

Figure 9. A Patient measuring his Heart Beat Rate.

Source: own elaboration. 
The Figures 8 and 9. shows that the patient is measuring his body temperature and heart beat rate by griping the corresponding sensors. His results can be viewed in the mobile phone, provided the Bluetooth $\left(\mathrm{HC}_{-} 05 / \mathrm{HC}_{-}\right.$06) should be paired with mobile on order to receive the data from device (Health care System).

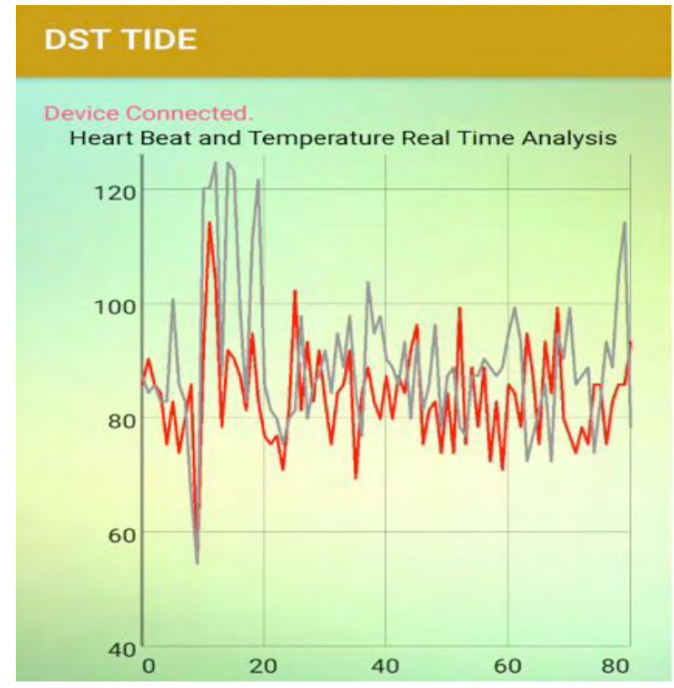

Figure 10. Temperature and Heart Beat Rate in Mobile.

Source: own elaboration.

The Figure 10 shows the graph of body temperature represented in Red Color and the reading is measured as $98.90 \mathrm{C}$ (latest value in the graph) whereas, the heart beat rate is represented in Grey Color and it is measured as 79 BPM (latest value in the graph) of a patient using a mobile phone. By using this device (Health Care System) vital signs (Temperature, Heart beat rate, Blood pressure, ECG, EEG) can be measured with corresponding sensors integrated to the single board computer which is programmed accordingly and can use the same this Android based Application (Mobile App) for monitoring the measurements. Even the Continuous Glucose Monitoring (CGM) can be measured with corresponding sensor, which measures the glucose level in blood of a human beings.

\section{CONCLUSIONS}

The Proposed architecture is designed based on extracting the various architectures by identifying the best one of each those architectures in order to meet the objective specified. The Bluetooth is used as transferring medium from a device to a Mobile app (Android 
Based mobile Application). It can able to transmit the data on real time for the vital signs measured in human body. This architecture is proposed for medical application, sensors are selected based on certain parameters as discussed in SECTION- II. The Bluetooth is preferred over other medium of data transmission because of its less energy consumption and secured data transfer over Zigbee, Wifi. In near future, this architecture can be used with many portable devices (Sensors) for measuring many more physical parameters of human body and even this architecture can be used for measuring Diabetes (Continuous Glucose Monitoring) according to the sensors used.

\section{ACKNOWLEDGEMENT}

We would like to thank International Research Center of Kalasalingam Academy of Research and Education for providing financial assistance under the scheme of University Research Fellowship(URF) and we also thank the Department of Electronics and Communication Engineering of Kalasalingam Academy of Research and Education, India for permitting to use the computational facilities available in Signal Processing and VLSI Design which was setup with the support of the Department of Science and Technology (DST).

\section{REFERENCES}

Acharya, U. R., Joseph, K. P., Kannathal, N., Lim, G. M., \& Suri, J. S. (2006). Heart rate variability: a review. Medical and biological engineering and computing, 44(12), 1031 1051. https://doi.org/10.1007/s11517-006-0119-0

Arefin, M. T., Ali, M. H., \& Haque, A. F. (2017). Wireless body area network: An overview and various applications. Journal of Computer and Communications, 5(7), 53-64. https://doi.org/10.4236/jcc.2017.57006

Asensio, A., Marco, A., Blasco, R., \& Casas, R. (2014). Protocol and architecture to bring things into internet of things. International fournal of Distributed Sensor Networks, 10(4), 158252. https://doi.org/10.1155/2014/158252

Atzori, L., Iera, A., \& Morabito, G. (2010). The internet of things: A survey. Computer Networks, 54(15), 2787-2805. https://doi.org/10.1016/j.comnet.2010.05.010 
Bandyopadhyay, S., Sengupta, M., Maiti, S., \& Dutta, S. (2011). Role of middleware for internet of things: A study. International Journal of Computer Science and Engineering Survey, 2(3), 94-105. http:/ / www.airccse.org/journal/ijcses/papers/0811cses07.pdf

Banuleasa, S., Munteanu, R., Rusu, A., \& Tonţ, G. (2016). IoT system for monitoring vital signs of elderly population. In 2016 International Conference and Exposition on Electrical and Power Engineering (EPE) (pp. 059-064). https://ieeexplore.ieee.org/ document/7781303

Beach, G., Krachunov, S., Pope, J., Fafoutis, X., Piechocki, R. J., Graddock, I., \& Casson, A. J. (2018). An ultra-low power personalizable wrist worn EGG monitor integrated with IoT infrastructure. IEEE Access, 6, 44010-44021. https:// doi.org/10.1109/ACGESS.2018.2864675

Deny,J., \& Sudharsan, R. R. (2020) Block Rearrangements and TSVs for a Standard Cell 3D IC Placement. In Peng, S. L., Son, L., Suseendran, G., \& Balaganesh, D. (eds.) Intelligent Computing and Innovation on Data Science. Lecture Notes in Networks and Systems, vol 118. Springer, Singapore. https://doi.org/10.1007/978-981-15-3284-9_24

Deny, J., Sudharsan, R. R., \& Kumaran, E. M. (2021). An orbicularis oris, buccinator, zygomaticus, and risorius muscle contraction classification for lip-reading during speech using sEMG signals on multi-channels. International fournal of Speech Technology. https://doi.org/10.1007/s10772-021-09816-0

Dixon, A. M., Allstot, E. G., Gangopadhyay, D., \& Allstot, D. J. (2012). Compressed sensing system considerations for ECG and EMG wireless biosensors. IEEE Transactions on Biomedical Circuits and Systems, 6(2), 156-166. https://doi.org/10.1109/ TBCAS.2012.2193668

Fatmi, H., Hussain, S., \& Al-Rubaie, A. (2017). Secure and cost-effective remote monitoring health-guard system. In 2017 IEEE Canada International Humanitarian Technology Conference (IHTC) (pp. 119-123). https://ieeexplore.ieee.org/ document/8058171 
Gubbi, J., Buyya, R., Marusic, S., \& Palaniswami, M. (2013). Internet of Things (IoT): A vision, architectural elements, and future directions. Future generation computer systems, 29(7), 1645-1660. https://doi.org/10.1016/j.future.2013.01.010

Hassanalieragh, M., Page, A., Soyata, T., Sharma, G., Aktas, M., Mateos, G., \& Andreescu, S. (2015). Health monitoring and management using Internet-ofThings (IoT) sensing with cloud-based processing: Opportunities and challenges. In 2015 IEEE International Conference on Services Computing (pp. 285-292). https:/ / doi. org/10.1109/SCG.2015.47

Kang, H. W., Kim, G. M., \& Koh, S.J. (2016). ISO/IEEE 1 1073-based healthcare services over IoT platform using 6LoWPAN and BLE: Architecture and experimentation. In 2016 International Conference on Networking and Network Applications (NaNA) (pp. 313-318). https://doi.org/10.1109/NaNA.2016.26

Kranjec,J., Beguš, S., Drnovšek,J., \& Geršak, G. (2013). Novel methods for noncontact heart rate measurement: A feasibility study. IEEE transactions on instrumentation and measurement, 63(4), 838-847. https://doi.org/10.1109/TIM.2013.2287118

Krishna, R. R., Kumar, P. S., \& Sudharsan, R. R. (2017). Optimization of wire-length and block rearrangements for a modern IC placement using evolutionary techniques. In 2017 IEEE International Conference on Intelligent Techniques in Control, Optimization and Signal Processing (INCOS) (pp. 1-4). https:/ /ieeexplore.ieee.org/document/8303081

Lama, S. (2017). Wireless Monitoring of Patients: Implementation of Wireless Body Area Network. https://www.theseus.fi/handle/10024/129373

Li, G., Hu, X., \& Zhang, L. (2017). The IoT-based heart disease monitoring system for pervasive healthcare service. Procedia computer science, 112, 2328-2334. https:/ / doi. org/10.1016/j.procs.2017.08.265

Misbahuddin, S., Zubairi, J. A., Alahdal, A. R., \& Malik, M. (2018). IoT-Based Ambulatory Vital Signs Data Transfer System. Journal of Computer Networks and Communications, 2018. Article ID 4071474. https://doi.org/10.1155/2018/4071474 
Peña-López, I. (2005). ITU Internet report 2005: the internet of things. https://www.itu.int/ $\mathrm{osg} / \mathrm{spu} /$ publications/internetofthings/

Prakash, R., Girish, S.V., \& Ganesh, A. B. (2016). Real-Time Remote Monitoring of Human Vital Signs Using Internet of Things (IoT) and GSM Connectivity. In Suresh, L., \& Panigrahi, B. (eds.) Proceedings of the International Conference on Soft Computing Systems. Advances in Intelligent Systems and Computing, vol 398. Springer, New Delhi. https://doi.org/10.1007/978-81-322-2674-1_5

Ren, Y., Werner, R., Pazzi, N., \& Boukerche, A. (2010). Monitoring patients via a secure and mobile healthcare system. IEEE Wireless Communications, 17(1), 59-65. https://ieeexplore.ieee.org/document/5416351

Siekkinen, M., Hiienkari, M., Nurminen, J. K., \& Nieminen, J. (2012). How low energy is bluetooth low energy? comparative measurements with zigbee/802.15.4. In 2012 IEEE wireless communications and networking conference workshops (WCNCW) (pp. 232-237). https:/ /ieeexplore.ieee.org/document/6215496

Sudharsan, R. R. (2019). Synthesis of FIR Filter using ADC-DAC: A FPGA Implementation. In 2019 IEEE International Conference on Clean Energy and Energy Efficient Electronics Circuit for Sustainable Development (INCCES) (pp. 1-3). https: / /ieeexplore.ieee. org/document/9167696

Sudharsan, R. R., \& Deny, J. (2020). Field Programmable Gate Array (FPGA)-Based Fast and Low-Pass Finite Impulse Response (FIR) Filter. In Intelligent Computing and Innovation on Data Science (pp. 199-206). Springer, Singapore.

Sudharsan, R. R., Deny, J., Kumaran, E. M., \& Geege, A. S. (2020). An Analysis of Different Biopotential Electrodes Used for Electromyography. Fournal of Nanoand Electronic Physics, 12(1), 01020-1 - 01020-7. https://jnep.sumdu.edu.ua/en/full_ article/2944

Sudharsan, R. R., Deny, J., Muthukumaran, E., \& Selvi, S. C. (2020). Design, implementation, and estimation of MFGV for 4-different position of human 
body using FPGA. Microelectronics fournal, 105, 104890. https://doi.org/10.1016/j. mejo.2020.104890

Sudharsan, R. R., Deny, J., Muthukumaran, E., \& Varatharajan, R. (2020). FPGA based peripheral myopathy monitoring using MFCV at dynamic contractions. Journal of Ambient Intelligence and Humanized Computing, 12, 7019-7027. https://doi. org/10.1007/s12652-020-02363-7

Swaroop, K. N., Ghandu, K., Gorrepotu, R., \& Deb, S. (2019). A health monitoring system for vital signs using IoT. Internet of Things, 5, 116-129. https://doi. org/10.1016/j.iot.2019.01.004

Tseng, G. H. (2013). Coordinator traffic diffusion for data-intensive Zigbee transmission in real-time electrocardiography monitoring. IEEE Transactions on Biomedical Engineering, 60(12), 3340-3346. https://doi.org/10.1109/tbme.2013.2266373

Wang, G., Atef, M., \& Lian, Y. (2018). Towards a continuous non-invasive cuffless blood pressure monitoring system using PPG: Systems and circuits review. IEEE Circuits and Systems Magazine, 18(3), 6-26. https://ieeexplore.ieee.org/document/8436482

Yang, Z., Zhou, Q., Lei, L., Zheng, K., \& Xiang, W. (2016). An IoT-cloud based wearable ECG monitoring system for smart healthcare. Fournal of medical systems, 40(12), 286. https://doi.org/10.1007/s10916-016-0644-9 
